BULLETIN OF THE

AMERICAN MATHEMATICAL SOCIETY

Volume 77, Number 2, March 1971

\title{
ON MEASURABILITY OVER PRODUCT SPACES ${ }^{1}$
}

\author{
BY R. M. DUDLEY
}

Communicated by M. H. Protter, June 29, 1970

The main result announced here is a negative solution of the Kakutani-Doob problem [3] on measurability of stochastic processes, assuming the continuum hypothesis. Thus the positive solution proposed earlier by M. Mahowald [6] is incorrect (the last step in the argument applies the Fubini theorem to sets in a product space which need not be measurable).

Complete proofs will appear in the Proceedings of the Sixth Berkeley Symposium on Mathematical Statistics and Probability. Preprints are available from the author.

The problem can be formulated as follows. Let

$$
x=\left(x_{t}(\omega), 0 \leqq t \leqq 1, \omega \in \Omega\right)
$$

be a real-valued stochastic process on $I=[0,1]$ over some probability space $(\Omega, P)$. Then by a theorem of Kolmogorov, $x$ has a probability distribution $P_{x}$ on the space $R^{I}$ of all functions from $I$ into the real line $R$. We embed $R$ in a compact space, such as its one-point compactification $\bar{R}$. Now $\bar{R}^{I}$ is also a compact Hausdorff space and $P_{x}$ defines a Baire measure on $\bar{R}^{I}$. We take the unique regular Borel extension $\vec{P}_{x}$ of $P_{x}$ (cf. Kakutani [5], E. Nelson [7]).

Let $E$ be the evaluation map $(t, f) \rightarrow f(t)$ from $I \times \bar{R}^{I}$ into $\bar{R}$, and let $\lambda$ be Lebesgue measure on $I$. Then the process $E(t, f)$ has the same probability laws as the original $x$, i.e. $P_{E}=P_{x}$, where $P_{E}$ is defined from $P_{x}$ as $P_{x}$ from $P$.

Thus we have a "canonical" representative from each class of processes $x$ with a given $P_{x}$. In general, $E$ need not be measurable for $\lambda \times \bar{P}_{x}$ [5]. The Kakutani-Doob problem asks: if $x$ is $\lambda \times P$-measurable, then is $E$ measurable for $\lambda \times \bar{P}_{x}$ ?

A negative answer, assuming the continuum hypothesis, can be given for certain processes of the form

$$
x(t, \omega)=\sum y_{n}(t) z_{n}(\omega)
$$

AMS 1970 subject classifications. Primary 28A35, 28A40, 60G05, 60G17; Secondary 60B05, 60G15, 02K25.

Key words and phrases. Product measure, Fubini theorem, measurable process, regular Borel measure, stochastic process, Gaussian process, white noise, continuum hypothesis.

1 This research was partially supported by National Science Foundation contract GP-14535. 
where the $y_{n}$ and $z_{n}$ are independent random variables, and $\sum y_{n}(t)$ and $\sum z_{n}(\omega)$ do not converge in general.

Perhaps the most interesting and important example of such a process is as follows. We replace $(I, \lambda)$ by an equivalent probability space $(H, \mu)$, where $H$ is a separable, infinite-dimensional real Hilbert space and $\mu$ is a suitable probability measure on $H$. We consider the standard Gaussian linear stochastic process $L$ on $H$. $L$ is a linear isometry from $H$ into Gaussian random variables with usual inner product. ( $L$ is the "white noise" or "isonormal" process.)

Specifically, let $H$ be the usual space $l_{2}$ of square-summable sequences $y=\left\{y_{n}\right\}_{n=1}^{\infty}$. Let $\mu$ be the probability on $H$ such that the $y_{n}$ are independent Gaussian with $E y_{n}=0$ and $E y_{n}^{2}=n^{-3 / 2}$. (Here it is important that $\sum n^{-3 / 2}<\infty$ and $\sum n^{-3 / 4}=\infty$; there are many other possibilities for $\mu$.) We have

$$
L(y)(\omega) \equiv \sum y_{n} G_{n}(\omega)
$$

where the $G_{n}$ are independent Gaussian random variables over some probability space $(\Omega, P)$ with $E G_{n} \equiv 0, E G_{n}^{2} \equiv 1$. Clearly $L$ is (jointly) measurable on $H \times \Omega$. Here is the main result:

Proposition 1. Assuming the continuum hypothesis, the set of $\lambda$-measurable functions from $H$ into $\bar{R}$ has $\bar{P}_{L}$-inner measure 0 . Hence

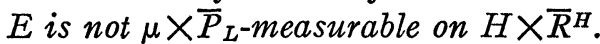

Most of the proof is in the following:

Lemma. Assuming the continuum hypothesis, suppose $A$ is a measurable subset of $\Omega$ with $P(A)>0$. Then there is a set $S \subset H$ with outer measure $\mu *(S)=1$ such that for every finite set $F \subset S$ and any nonempty open sets $U_{f} \subset R$,

$$
P\left\{\omega \in A: L(f)(\omega) \in U_{f} \text { for all } f \in F\right\}>0 .
$$

The construction of $S$ by transfinite induction follows a method suggested by Sierpinski's proof [8] that there is a set in the plane which intersects every line in at most two points yet is not Lebesgue measurable.

Bledsoe and Morse [2] extend a usual product measure $\nu \times \rho$ to a measure $\nu X_{M} \rho$ by setting $\left(\nu \times_{M} \rho\right)(B)=0$ if and only if

$$
\iint \chi_{B}(x, y) d \nu(x) d \rho(y)=\iint \chi_{B}(x, y) d \rho(y) d \nu(x)=0 .
$$

This gives Sierpiński's set measure 0 in the plane. But in our case, $E$ is not measurable even for $\mu \times_{M} \bar{P}_{L}$. 
To prove Proposition 1 given the Lemma, suppose $C$ is a compact set in $\bar{R}^{H}$ with $\bar{P}_{L}(C)>0$. We take a Baire set $C_{1} \supset C$ with the same measure. Then $C_{1}$ is equivalent to a set $A \subset \Omega$. Applying the Lemma to $A$ shows that all functions from $S$ into $\bar{R}$ extend to functions in $C$, so that not all functions in $C$ can be measurable. Proposition 1 follows directly.

The other results to be presented now have proofs independent of Proposition 1 and its proof. They do, however, help confirm that product spaces such as $\bar{R}^{I}$ are rather pathological for measure theory. In what follows, convergence of Borel probability measures $P_{n} \rightarrow P_{0}$ on a topological space means the usual (weak, weak-star, narrow) convergence: $\int f d P_{n} \rightarrow \int f d P_{0}$ for every bounded continuous real $f$.

Proposition 2. There exist processes $x(n)$ such that each $\bar{P}_{x(n)}$ is concentrated in a finite set of functions, so that $E$ is $\lambda \times \bar{P}_{x(n)}$-measurable for all $n$, and such that $\bar{P}_{x(n)} \rightarrow Q$ for some $Q$, but $E$ is not $\lambda \times Q$-measurable.

To prove Proposition 2, we can take $Q=P_{v}$ where the $v(t)$ are independent for different $t \in I$ and $P(v(t)=0)=P(v(t)=1)=1 / 2$.

Proposition 3. There are continuous functions $f_{n}$ from $I$ into $I^{I}$ with product topology such that $f_{n}(x)(y) \rightarrow f(x)(y)$ for all $x$ and $y$ but $f$ is not measurable from $(I, \lambda)$ into $I^{I}$ with its Borel $\sigma$-algebra.

Proof. Let $f_{n}(x)(y)=\max (0,1-n|x-y|)$. Then $f(x)(y)=1$ if $x=y$ and 0 otherwise, so $f$ is not measurable. Q.E.D.

A topological space $(S, \Im)$ is called normal if for any disjoint closed sets $F, G$ there are disjoint open sets $U, V$ with $F \subset U, G \subset V .(S, J)$ is called perfectly normal iff it is normal and every open set is a countable union of closed sets.

Proposition 4. If $f_{n}$ are measurable functions from a measurable space $(X, \S)$ into a perfectly normal topological space $(S, J)$ such that $f_{n}(x) \rightarrow f(x)$ for all $x \in X$, then $f$ is measurable.

Propositions 3 and 4 correct a result of Fernique [4, Théorème I, 4.2(a) ]. Fernique shows that many useful spaces in addition to metric spaces are perfectly normal [4, Théorème I, 6.1, p. 19]. Such spaces include the "Lusin spaces" which are 1-1 continuous images of complete separable metric spaces and are regular topological spaces.

But even Lusin spaces turn out not to have all the good properties of metric spaces, such as Skorohod's theorem [9, Theorem 3.1.1, p. 281] that if probabilities converge, $P_{n} \rightarrow P_{0}$, over a (complete) separable metric space, then there exist random variables $X_{n}$ with 
distributions $P_{n}$ and $X_{n} \rightarrow X_{0}$ almost surely. The following example is based on one of Fernique's (cf. Badrikian [1, Exposé 8, No. 6]).

Proposition 5. There exist Borel probability measures $\mu_{n}$ on a Hilbert space $H$ with $\mu_{0}$ concentrated at 0 such that $\mu_{n} \rightarrow \mu_{0}$ for the weak topology on $H$ but there are no random variables $X_{n}$ with distributions $\mu_{n}$ which converge weakly to 0 with positive probability.

To prove this, let $\left\{\varphi_{m}\right\}$ be an orthonormal basis of $H$ and let $\mu_{n}$ give mass $1 / 2^{n}$ to $n \varphi_{m}$ for $m=1, \cdots, 2^{n}$.

Acknowledgement. I am indebted to C. L. Reedy for contributing Proposition 4 and an earlier result in the direction of Proposition 3.

\section{REFERENCES}

1. Albert Badrikian, Séminaire sur les fonctions aléatoires linéaires et sur les mesures cylindriques, Lecture Notes in.Math., no. 139, Springer-Verlag, Berlin and New York, (1970).

2. W. W. Bledsoe and A. P. Morse, Product measures, Trans. Amer. Math. Soc. 79 (1955), 173-215. MR 16, 1008.

3. J. L. Doob, Probability in function space, Bull. Amer. Math. Soc. 53 (1947), 15-30. MR 8, 472.

4. Xavier Fernique, Processus linéaires, processus généralisés, Ann. Inst. Fourier (Grenoble) 17 (1967), fasc. 1, 1-92. MR $36 \# 4628$.

5. Shizuo Kakutani, Notes on infinite product spaces. II, Proc. Imp. Acad. Tokyo 19 (1943), 184-188. MR 7, 279.

6. Mark Mahowald, On the measurability of stochastic processes, Illinois J. Math. 8 (1964), 312-315. MR 28 \#5464.

7. Edward Nelson, Regular probability measures on function space, Ann. of Math. (2) 69 (1959), 630-643. MR 21 \#4479.

8. Waclaw Sierpiński, Sur un problème concernant les ensembles mesurables superficiellement, Fund. Math. 1 (1920), 112-115.

9. A. V. Skorohod, Limit theorems for stochastic processes, Teor. Verojatnost i Primenen 1 (1956), 289-319=Theor. Probability Appl. 1 (1956), 261-290. MR 18, 943.

Massachusetts Institute of Technology, Cambridge, Massachusetts 02139 